\title{
TOWARDS A REALISTIC MODEL FOR NOVA ENVELOPES
}

\author{
M. FRIEDJUNG \\ Institut d'Astrophysique, Paris
}

\begin{abstract}
Arguments are given, suggesting that the most probable type of model is one in which ejection continues after maximum light in the visual region, but with rapid time variations.
\end{abstract}

There is still much uncertainty and controversy concerning the structure of the envelopes ejected by novae and the nature of the ejection which produces them. Several very different schools of thought exist, between which there is much disagreement and little communication. Here I shall attempt to briefly summarize the situation, and to indicate the direction in which a solution to these problems may perhaps be found.

The types of ejection models suggested can be very broadly classified as shown in the following sections.

\section{Instantaneous Ejection}

In such models, all or nearly all the envelope is ejected in a time which is short compared with that in which a nova undergoes its characteristic development after maximum light. Two simple forms of this type of model exist (see Friedjung, 1968):

Instantaneous ejection type I. Most of the envelope is ejected at the same velocity and is in a thin shell. This very simple type of model was extensively studied by Pottasch (1959).

Instantaneous ejection type II. The ejection velocity of different parts of the envelope is not the same. The result is that instead of remaining in a thin shell the ejected gas fills a larger volume with the gas with the largest velocity at the outer edge. A model of this type has recently been proposed by Nariai (1974), but the idea is much older.

\section{Continued Ejection}

In models of this type, ejection continues after the visual maximum, but generally tends to decrease with time. In the simplest situation that can be envisaged two density maxima then occur in the envelope. One maximum is due to gas ejected when the ejection rate was high in the early phases, and occurs near the outer edge of the envelope. The other occurs near the central star, where the newly ejected gas has not expanded much. Two simple forms of continued ejection models can be distinguished (see Friedjung, 1966).

Continued ejection A. The density maximum near the central star contributes most of the observed light. It is this sort of model, which I.have supported in my papers. 
Continued ejection $B$. The density maximum near the outer edge of the envelope contributes most the observed light. The properties of this sort of model are similar to those of instantaneous ejection type $I$.

\section{Central Star Dominant}

According to this kind of model, most of the light observed of a nova (at least of the continuous spectrum) comes from the central star, parts of which may have expanded considerably. A model of this nature is supported by Mustel (1957).

Most of these models are, at least in their simple forms, not compatible with observation. There are difficulties in interpreting light curves; thus the rate of visual fading of nova V603 Aquilae between the phases when it is the most certain that the hydrogen was almost completely ionized, was slower than what would be expected according to instantaneous ejection type I or continued ejection B, unless the ejected envelope is assumed to have been decelerated or to have increased in volume more slowly than in the simplest forms of these models. The recent results of far UV observations (Gallagher and Code, 1974), showing that the bolometric luminosity can remain constant during the early stages of visual fading, increase these problems.

A study of the spectra gives more precise arguments. It must first be noted that there are good reasons to believe that the ejection velocity is not the same in different parts of the envelope and that the parts with higher velocities in the phases between (visual) maximum light and the nebular stage are nearer the central star than those with lower ejection velocities (McLaughlin, 1964). The regions with higher ejection velocities tend to appear later than those with lower velocities, implying later ejection. Furthermore an analysis of the spectra in certain cases, indicates that a large part of the continous spectrum is at least sometimes produced near the centre of the envelope. Thus the narrowness of the principal system of absorption components of nova V603 Aquilae produced by relatively low-velocity gas probably near the outer edge of the envelope is, when studied in detail, difficult to understand unless much of the continuous spectrum was formed much nearer the centre of the envelope. (This conclusion may perhaps also be drawn for the region of emission-line formation, but this is much less certain.) Indeed a large part of the continuous spectrum of this nova was, at least in certain phases, formed even nearer the centre of the envelope than the Orion absorption components, which were produced by gas at a higher velocity than that of the principal system and nearer the centre. This conclusion follows from the strength of line absorption due to N III. Similarly a study of nova Delphini 1967 (Friedjung and Malakpur, 1974) indicates that the continuous spectrum was produced in a region much smaller than the total size of the envelope during the phase before the maximum of December 1967. However, the argument in this last case is unfortunately not watertight. These spectroscopic results are not easily compatible with instantaneous ejection type I or continued ejection $\mathbf{B}$.

Observations of spectra also seem to contradict instantaneous ejection type II in most cases. In models of this type, the ejection velocity would be smallest near the 
central star, which directly contradicts the result given above for novae after maximum visual brightness. (It should however be noted that these arguments cannot be applied to recurrent novae, similar to RS Ophiuchi and T Coronae Borealis.) In the case of central star dominant models, one would expect to see in many phases unshifted narrow lines formed in a stationary layer near the central star. Such lines are generally not observed, while the narrow emission lines observed in the spectrum of the recurrent nova RS Ophiuchi can be very convincingly explained as being due to a stationary circumstellar cloud (Pottasch, 1967).

This process of elimination leaves, at least for some novae, only continued ejection A among the simple models. There are, however, a number of serious difficulties with simple forms of this kind of model. It is reasonable to suppose that the continued ejection is of high-velocity gas producing the Orion absorption system, the lower-velocity gas responsible for the principal absorption system being ejected near visual maximum. This conclusion follows from what has already been said about velocity stratification. In this case, collisions could be expected between the higher and lower velocity gas, and the latter could be expected to be accelerated unless it was much more massive. Now large accelerations of the principal absorption system are not observed for most novae, this being especially true if the phase immediately following the visual maximum is not considered. Also the line emission in the nebular stage, when most of the activity has ceased, is produced by gas having the same expansion velocity as that of the principal absorption system, while nova V603 Aquilae, which was in certain ways typical of many novae, had also in earlier phases of its development most of its line emission produced by gas with the principal system expansion velocity. These results are difficult to understand if simple ejection rate variation calculations are performed assuming continued ejection $\mathrm{A}$ and grey extended atmosphere models based on the classical theory of Kosirev.

To overcome these difficulties, one can always hope that good extended atmosphere models taking into account non-LTE effects would, when combined with the observations, give much more rapid rates of decrease of the ejection rate with time. In spite of recent work in this field, suitable models do not yet exist. One can also invent somewhat artificial hypotheses such as the deceleration of the higher velocity gas to the principal system absorption velocity, or continued ejection with the latter velocity. Such hypotheses (one of which was proposed by me some years ago (Friedjung, 1968)) would not be easy to reconcile with the observations, as well as not being very elegant.

A relatively straightforward method of at least partly resolving these problems is to suppose rapid ejection rate variations, so that the thickness in the radial direction of the masses of gas containing most of what is ejected after the visual maximum, is much smaller than the distance from the central star. Thus if the thickness in the radial direction is $T$, and the mass absorption coefficient is proportional to the density, it can be easily shown that, if a mass of gas is supposed to become optically thick at a known distance from the central star, the mass required is proportional to $\sqrt{T_{0}}$. This means that the total mass required and hence the ejection rate required to produce a photosphere in the ejected envelope at a certain distance from the central star, and 
hence a certain brightness of the continuous spectrum, can be reduced if $T$ is reduced. The factor by which the required ejection rate is reduced can become very large if the thermal diffusion of gas in the radial direction is inhibited by a magnetic fied whose effect would become important if it was more than $10 \mathrm{G}$ in the regions producing the continuous spectrum.

It is not so easy to see how the line emission of the region producing the Orion absorption system could be reduced. If the problem is not solvable by detailed line transfer calculations, it might be necessary to propose a more complex model. A solution would be if the expansion of the masses of gas already discussed was inhibited in all directions by a magnetic field so that they did not become optically thin before being destroyed by collisions with slower moving gas. They would be expected to cool and become invisible before this happened, becoming also separated and unable to absorb much radiation from below.

What I have suggested is tentative and uncertain. It may perhaps overcome the contradictions and combine the advantages of continued ejection $\mathrm{A}$ and instantaneous ejection type I. The consistency of the results of Pottasch (1959) obtained assuming the latter model would be simply explained, because most of the mass of the envelope would be due to the gas ejected near the visual maximum. If these suggestions are close to the truth, rapid line profile variations with time scales of the order of a few hours might be expected. It would be useful to look for such variations, and to study the profiles in detail. It is also important to direct theoretical work to the explanation of such forms of ejection.

\section{References}

Friedjung, M.: 1966, Monthly Notices Roy. Astron. Soc. 131, 447.

Friedjung, M.: 1968, Astrophys. Letters 2, 121.

Friedjung, M. and Malakpur, I.: 1974, Astrophys. Space Sci. 25, 433.

Gallagher, J. S. and Code, A. D.: 1974, Astrophys. J. 189, 303.

McLaughlin, D. B.: 1964, in Novae, Novoides et Supernovae, Centre National de la Recherche Scientifique, Paris, p. 123.

Mustel, E. R.: 1957, in G. H. Herbig (ed.), 'Non-Stable Stars', IAU Symp. 3, 57.

Nariai, K.: 1974, Astron. Astrophys. 36, 231.

Pottasch, S. R.: 1959, Ann. Astrophys. 22, 394.

Pottasch, S. R.: 1967, Bull. Astron. Inst. Neth. 19, 227. 